\title{
Breast metastasis from a malignant melanoma- a
}

\section{case report}

\begin{abstract}
Metastatic lesions in the breast from malignant diseases originated in other primary sources are rare. Mostly arise from the contra-lateral breast. The incidence extramammary origin of the breast metastasis is $1.2-2 \%$. The usual extra-mammary primary sites of metastasis are malignant melanoma, lung cancer and Non-Hodgkin lymphoma. In any patient who has been diagnosed with a known primary cancer, if presented with a breast lump, a metastatic breast focus should be considered. The malignant melanoma $[\mathrm{MM}]$ characterised by widespread dissemination but infrequently metastasizes to the breast. In this paper we report a case of 43 years old female with malignant melanoma with breast metastases.
\end{abstract}

Keywords: malignant diseases, non-hodgkin lymphoma, malignant melanoma, contra-lateral breast
Volume 3 Issue 3 - 2017

\author{
A Saad Abdalla Al-Zawi,' K Osayi, ${ }^{2}$ Miss \\ Jessica Eades ${ }^{3}$ \\ 'Breast \& General Surgeon, Basildon \& Thurrock University \\ Hospital, UK \\ ${ }^{2}$ Consultant Histopathologist, Basildon \& Thurrock University \\ Hospital, UK \\ ${ }^{3}$ Diagnostic Radiography, Basildon \& Thurrock University \\ Hospital, UK
}

\begin{abstract}
Correspondence: A Saad Abdalla Al-Zawi, Breast \& General Surgeon, Basildon University Hopsital, Nerthermayne Basildon ESSECx SSI6 5NL, Tel 77I0605।40,

Email abdalasaad@gmail.com
\end{abstract}

Received: June 17,2017 | Published: June 27, 2017

\section{Introduction}

It is rarely for the breast to harbour a malignant metastatic disease. Breast metastatic masses arise often from a primary cancer of the contra-lateral breast. Breast secondary cancer from extramammary malignancies is even rarer. $^{1}$ In 1903 metastatic chordoma of the breast, was reported by Trevithick. ${ }^{2}$ In contrast to primary breast tumours, it accounts for approximately $2 \%$ of breast tumours. ${ }^{3}$ In addition to hematopoietic malignancies; pulmonary cancer, melanoma and gastric carcinoma are most repeatedly described as primary cancer sites to metastasize to the breast. ${ }^{4}$ Breast melanoma potentially can be primary in origin. The breast metastatic melanoma can be presented as a solitary lesion or as multiple unilateral or bilateral masses. These lesions could be palpable clinically or just incidental findings on imaging. ${ }^{5}$ We present a case of melanoma with metastasis to the breast in a pre-menopausal female patient.

\section{Case report}

A 43years old white premenopausal female patient admitted though Emergency Department with a recent history of unwell feeling in addition to sudden onset of dizziness, ataxia, vomiting \& loss of vision on right eye. Initial clinical assessment revealed loss of coordination, as well as blind right eye and partial unilateral deafness. The patient partner pointed to a smaller pigmented nevus in the anterior chest wall which started to be darker recently. Clinically, there is bilateral indeterminate scattered breast modularity and a small breast lump at the outer upper quadrant of the right breast measures about $30 \mathrm{~mm}$. Mammogram and breast US showed bilateral scattered irregular hypo echoic suspicious lesions measures between $5 \mathrm{~mm} \&$ 20mm, graded as M4U4. [BI-RADS: Breast Imaging Reporting and Data System]. A core biopsy was taken from the largest lesion in the right breast (Figure 1) (Figure 2).

CT\& MRI of the brain revealed multiple rounded enhancing masses scattered through the brain and are likely to be cerebral metastases. One lesion in left cerebellar lesion showed a little high signal in the CT head \& brain MRI, this feature is a characteristic for haemorrhagic phenomena or presence of melanin in melanoma metastasis (Figure 3).
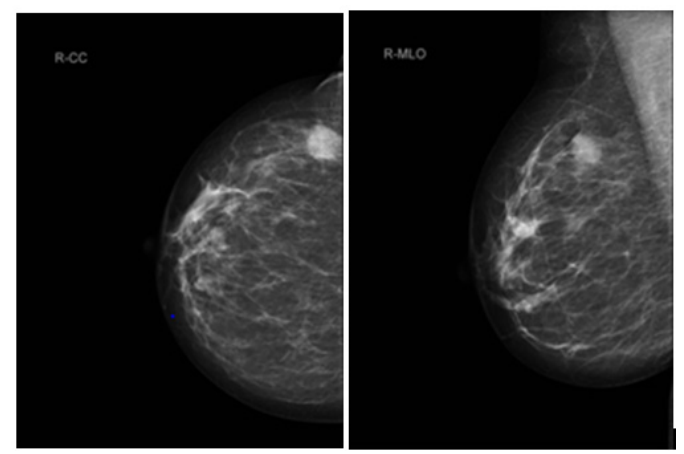

Figure I A, CC view, B, MLO view.

CC (cranial-caudal) \& MLO (medio-lateral-oblique) views of right mammogram indicating $17 \mathrm{~mm}$ suspicious lesion in the OUQ (outer upper quadrant).

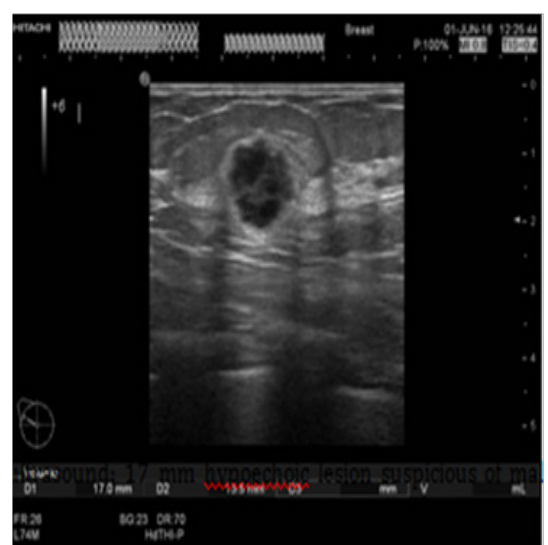

Figure 2 Beast ultrasound: $17 \mathrm{~mm}$ hypoechoic lesion suspicious of malignancy at 10 o'clock position of right breast. 


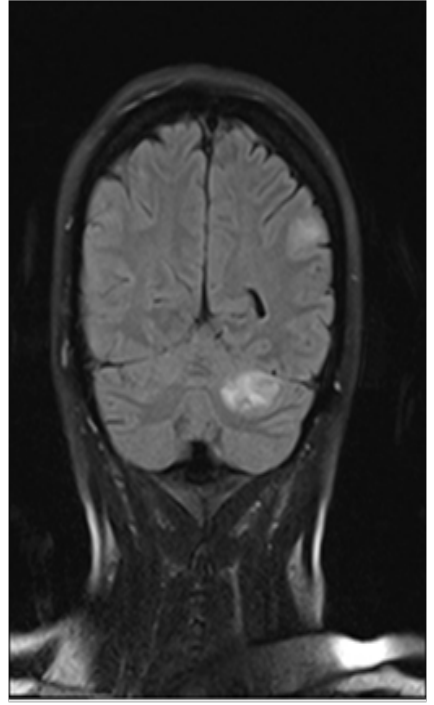

Figure 3 Head MRI, Left cerebellar lesion with a high signal on TI sequence.

Staging CT chest \& abdomen, showed bilateral lung nodules and numerous subcutaneous nodules, the largest seen in the umbilical area (Figure 4). Histology of the right breast biopsy reported staining for Melan-A \& HMB45 is positive (Figures 5A-5D). No staining for Cam5.2, ER, PR, CK7 and CK20. Ki67 is 10\%. Appearances and immuno-profile are consistent with metastatic malignant melanoma. Due to disease dissemination, the patient has been referred to palliative oncological treatment.

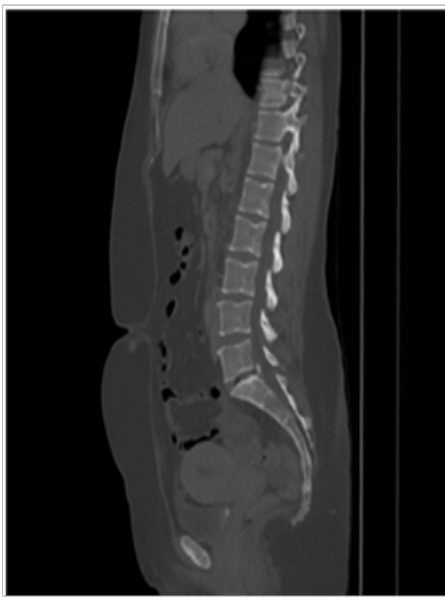

Figure $4 \mathrm{CT}$ abdomen with a subcutaneous nodule in the umbilical area.

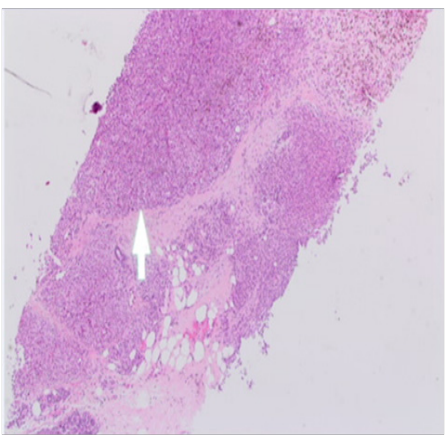

Figure 5A $\times 2$ magnification, normal breast lobules at 7'o clock, sheets of melanoma at the centre and necrosis with melanin pigment at l'o clock.

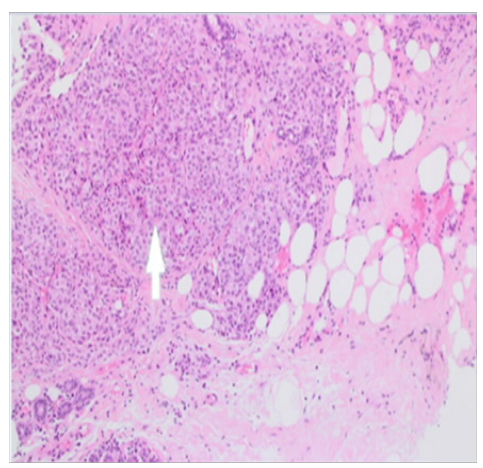

Figure 5B $\times 4$ magnification, normal breast lobules at 7'o clock, sheets of melanoma at the centre and necrosis with melanin pigment at l'o clock.

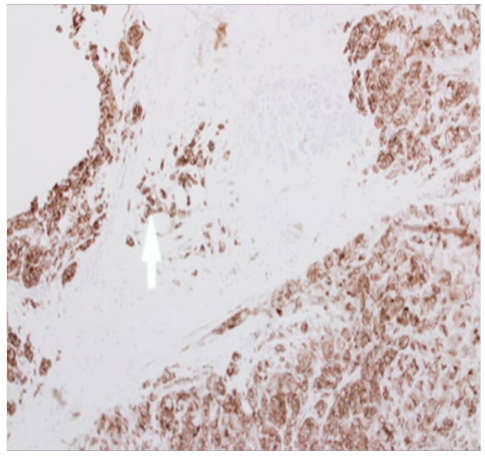

Figure 5C x10 sheets of melanocytes positive for HMB45. Normal breast lobules at 12 o'clock do not stain for HMB45.

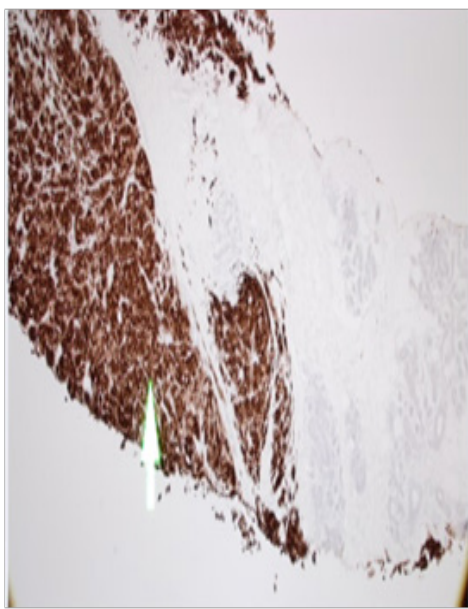

Figure 5D $\times 40$ sheets of melanocytes on the left are positive for Mel A Normal breast lobules on the right do not stain for Mel A.

\section{Discussion}

Breast melanoma could arise basically in the breast skin, primarily in breast parenchyma, metastases to breast tissue, in-transit metastases (IMs) to breast glandular tissue or metastases to the breast skin. ${ }^{6}$ Metastatic breast lesions might be the first presentation clinical sign of the primary malignancy. ${ }^{7}$ Breast metastatic lesions from extramammary origin are uncommon. They encounter only $2 \%$ of total breast tumours. $^{8}$

The top three sites are the contra-lateral breast, lymphomas and disseminated melanoma. Other sites are ovarian, lung, and gastric malignancies in addition to carcinoids \& rhabdomyosarcomas. ${ }^{9}$ 
In males, it has been reported that prostate adenocarcinoma can metastasise to the breast. ${ }^{10}$ The evidence showed that, the incidence of melanoma has been steadily rising in the recent decades, with an annual increase of $38 \%$ worldwide and the most common forms are cutaneous and ocular. ${ }^{11}$ Melanoma can metastasise to lymph nodes, other skin areas, and distant organs such as the liver, lung, brain, bones $\&$ breast. $^{12}$ The majority of primary melanoma in pre-menopausal females is the lower extremities; however, the most common primary sites for melanoma in pre-menopausal women with breast metastatic lesions are the trunk and upper limbs. ${ }^{13}$ The lateral aspect of the breast, frequently reported as the site of the metastasis, this explained by the good vascularity and the presence of more glandular tissue. It has been reported that, in about $50 \%$ of cases, the upper outer quadrant was involved. ${ }^{9}$ The metastatic tumours to the breast are frequently found to be superficial, not attached to the surrounding tissue with or without skin involvement. They are usually firm well circumscribed masses. ${ }^{14}$ The bilateral breast involvement with MM [Metastatic melanoma] represents about $10 \%$ of patients who has breast metastases from melanoma; this indicates a possibility of a widely disseminated disease which is poor prognostic factor. ${ }^{15}$

The case presented in this paper is of a wide spread disease, diagnosed initially from breast lesion biopsy. The sample was positive for Melan-A [melanoma antigen can be found on the surface of melanoma cells].

Also was positive for HMB45, which is a monoclonal antibody that reacts against an antigen present in melanomas. The specific antigen recognized by HMB-45 is now known as Pmel-17. No staining was detected for estrogens and progesterone receptors. It was negative for CK7 \& CK20 which are characteristic markers for the glandular epithelia. Also was negative for Cam5.2 which is, anti-Cytokeratin stains most epithelial-derived tissue, including liver, renal tubular epithelium, and hepatocellular and renal cell carcinomas.

\section{Conclusion}

Breast metastasis from extra-mammary malignancy is a very rare in occurrence. Metastasis should be considered if the patient presented with a history of a known primary malignancy, however this may happen even without a previous history. The immuno-histochemical stains should always be performed. Generally speaking, in a scenario, as in our case, these lesions reflect wide disseminated disease. This will have an impact on the prognosis assessment, which usually is extremely unfavourable.

\section{Acknowledgements}

None.

\section{Conflict of interest}

Author declares that there is no conflict of interest.

\section{References}

1. Williams SA, Ehlers RA, Hunt KK, et al. Metastases to the breast from nonbreast solid neoplasms: presentation and determinants of survival. Cancer. 2007;110(4):731-737.

2. Edgar Trevithick. A case of chloroma with clinical history and account of post mortem appearances. Lancet. 1903;2:158-160.

3. Youyeon Kim, Kyu Ran Cho, Bo Kyoung Seo, et al. Radiologic Findings of Metastatic Malignant Melanoma of the Breast: Mammographic, Sonographic, Dynamic Contrast-Enhanced Breast MRI, and 18F-FDG PET-CT Features. Iranian Journal of Radiology. 2016. p. e38392.

4. Drueppel D, Schultheis B, Solass W, et al. Primary malignant melanoma of the breast. Anticancer Res. 2015;35(3):1709-1713.

5. Nataša Prvulović, Dragana Đilas-Ivanović, Borislava Nikolin, et al. Melanoma metastatic to the breast: a report of an unusual case. Arch Oncol. 2011;19(3-4):79-80.

6. Kurul S, Taş F, Büyükbabani N, et al. Different manifestations of malignant melanoma in the breast: a report of 12 cases and a review of the literature. Jpn J Clin Oncol. 2005;35(4):202-206.

7. Leonardo Solaini, Anna Bianchi. A Mammary Nodule Mimicking Breast Cancer. Int Surg. 2005;99(3):200-202.

8. Ioannidis Charilaos. Melanoma metastatic to the breast. An Obstetric \& Gynaecoogy International Journal. 2014;13(4):122-126.

9. Moschetta M, Telegrafo M, Lucarelli NM, et al. Metastatic breast disease from cutaneous malignant melanoma. Int J Surg Case Rep. 2014;5(1):34-36.

10. Drelichman A, Amer M, Pontes E, et al. Carcinoma of prostate metastatic to breast. Urology. 1980;16(3):250-255.

11. Yang B, Li Q2, Zhao H, et al. Local anaesthetic thoracoscopy for the diagnosis of metastatic pleural melanoma originated from oral malignant melanoma. World J Surg Oncol. 2015;13:326.

12. Yeu-Tsu N (Margaret) Lee. Malignant melanoma: Pattern of metastasis. CA-A Cancer Journal for Clinicians. 1980;30(3):137-142.

13. Al Samaraee A, Khout H, Barakat T, et al. Breast Metastasis From a Melanoma. Ochsner J. 2012;12(2):149-151.

14. Gulistan Bahat. Melanoma metastasis to the breast: A diagnostic pitfall. Cancer Detect Prev. 2009;32(5-6):458-461.

15. Majeski J. Bilateral breast masses as initial presentation of widely metastatic melanoma. J Surg Oncol. 1999;72(3):175-177. 